\title{
Role of internal motions and molecular geometry on the NMR relaxation of hydrocarbons
}

\author{
P. M. Singer, D. Asthagiri, Z. Chen, A. Valiya Parambathu, G. J. Hirasaki, \\ and W. G. Chapman \\ Department of Chemical and Biomolecular Engineering, Rice University, 6100 Main St., Houston, \\ Texas 77005, USA
}

(Received 22 January 2018; accepted 11 April 2018; published online 30 April 2018)

\begin{abstract}
The role of internal motions and molecular geometry on ${ }^{1} \mathrm{H}$ NMR relaxation rates in liquid-state hydrocarbons is investigated using MD (molecular dynamics) simulations of the autocorrelation functions for intramolecular and intermolecular ${ }^{1} \mathrm{H}-{ }^{1} \mathrm{H}$ dipole-dipole interactions. The effects of molecular geometry and internal motions on the functional form of the autocorrelation functions are studied by comparing symmetric molecules such as neopentane and benzene to corresponding straight-chain alkanes $n$-pentane and $n$-hexane, respectively. Comparison of rigid versus flexible molecules shows that internal motions cause the intramolecular and intermolecular correlation-times to get significantly shorter, and the corresponding relaxation rates to get significantly smaller, especially for longer-chain $n$-alkanes. Site-by-site simulations of ${ }^{1} \mathrm{H}$ 's across the chains indicate significant variations in correlation times and relaxation rates across the molecule, and comparison with measurements reveals insights into cross-relaxation effects. Furthermore, the simulations reveal new insights into the relative strength of intramolecular versus intermolecular relaxation as a function of internal motions, as a function of molecular geometry, and on a site-by-site basis across the chain. Published by AIP Publishing. https://doi.org/10.1063/1.5023240
\end{abstract}

\section{INTRODUCTION}

Recent studies have shown that MD (molecular dynamics) simulations can successfully predict ${ }^{1} \mathrm{H}$ NMR (nuclear magnetic resonance) relaxation rates $R_{1,2}$ and diffusion coefficients of liquid-state hydrocarbons and water, without any adjustable parameters in the interpretation of the simulation data. ${ }^{1}$ Besides validating the force fields used in the simulations, the MD simulations reveal new insights about the NMR relaxation from intramolecular versus intermolecular ${ }^{1} \mathrm{H}-{ }^{1} \mathrm{H}$ dipole-dipole interactions in fluids, which are not easily accessible experimentally. ${ }^{2}$ Specifically, the simulations quantify the relative strength of the two relaxation mechanisms, indicating that intramolecular relaxation increasingly dominates over intermolecular relaxation with increasing molecular chain-length (i.e., increasing carbon number). This justifies the common practice of only considering intramolecular dipole-dipole interactions for simulations of macromolecules such as proteins ${ }^{3}$ or polymers in the short-time regime. $^{4}$

As also reported in Ref. 1, the functional forms of the simulated autocorrelation functions for intramolecular $G_{R}(t)$ and intermolecular $G_{T}(t)^{1} \mathrm{H}-{ }^{1} \mathrm{H}$ dipole-dipole interactions show deviations from the traditional models by Bloembergen, Purcell, Pound (BPP) ${ }^{5}$ Torrey (TOR), ${ }^{6}$ and Hwang and Freed (HF). ${ }^{7}$ In the case of intramolecular $(R)$ interactions, the BPP model predicts a single-exponential decay for $G_{R}(t)$ with rotational correlation-time $\tau_{R}$; however, the simulations clearly indicate an increasingly "stretched" (i.e., multi-exponential like) decay with increasing chain-length. For intermolecular $(T)$ interactions, the TOR and HF models predict a specific functional form for $G_{T}(t)$ (and an associated translational correlation-time $\tau_{T}$ ); however, the simulations also indicate a stretched decay at large chain-lengths. Another prediction from the traditional models ${ }^{5-7}$ is that the ratio of translationaldiffusion correlation-time $\tau_{D}{ }^{8}$ to rotational-correlation time $\tau_{R}$ should be $\tau_{D} / \tau_{R}=9$ according to the Stokes-Einstein relation $^{9}$ for diffusion of spherical molecules through a liquid with low Reynolds numbers. However, the simulations clearly show that $\tau_{D} / \tau_{R} \ll 9$ at large chain-lengths, ${ }^{1}$ which is most likely due to the presence of internal motions for long-chain molecules.

Simplified models for the autocorrelation function of non-spherical and non-rigid molecules have been successfully developed in the past, such as those by Woessner and others which describe spin-relaxation processes in twoproton systems undergoing anisotropic reorientation ${ }^{10-12}$ and internal motions, ${ }^{13}$ as well as three-proton systems. ${ }^{14}$ These anisotropic reorientation models have been successful for highly symmetric molecules such as benzene, where in-plane and perpendicular-to-plane rotational diffusion-coefficients (and rotational correlation-times) can be determined from NMR measurements and MD simulations. ${ }^{15,16}$ In the case of intramolecular (i.e., rotational) relaxation for molecules, such theories predict a multi-exponential decay for the autocorrelation function $G_{R}(t)$, with a corresponding distribution in correlation times. Generalizations of such models for the autocorrelation function later arose which take a phenomenological approach to the internal motions, such as the LipariSzabo model ${ }^{17,18}$ often used in polymers ${ }^{19}$ and proteins. ${ }^{20}$ However, as the internal motions of the molecule become more complex, one is invariably forced to develop even more 
phenomenological models of the autocorrelation function and its associated spectral density. ${ }^{21,22}$ Such phenomenological approaches include the Cole-Davidson distribution function, ${ }^{4,23}$ the generalized gamma function, ${ }^{19}$ the KohlrauschWilliams-Watts ${ }^{4}$ functions, and the Singer-Hirasaki function, ${ }^{24,25}$ each chosen to fit the observations but without theoretical justification. In the case of bulk water (which exhibits hydrogen-bonding), $G_{R}(t)$ is stretched to a similar extent as $n$-pentane, ${ }^{1}$ which may be a result of large, discrete angular-jumps ${ }^{26}$ superimposed on a continuous-time rotational-diffusion process. ${ }^{27}$

Important insights into the molecular dynamics and dipolar couplings of hydrocarbons have previously been studied using ${ }^{2} \mathrm{H}$ NMR spectroscopy and relaxometry of partially deuterated hydrocarbons oriented in liquid-crystal solvents, ${ }^{28-36}$ which do not require any phenomenological models. In such systems, the spectral densities, residual dipolarcouplings, and internal motions of hydrocarbons oriented in liquid-crystal solvents can be measured as a function of temperature on a site-by-site basis across the hydrocarbon chain. More specifically, the ${ }^{2} \mathrm{H}$ NMR of hydrocarbons allows for studying the effects of internal motions on intramolecular dynamics on a site-by-site basis across the chain and could in principle be compared to the results presented here, although this is beyond the scope of this work. More recently, the conformations of $n$-alkanes oriented in liquid-crystal solvents have been measured using ${ }^{1} \mathrm{H}$ NMR spectroscopy as a function of temperature and compared with MD simulations. ${ }^{37-39}$ While oriented hydrocarbons in liquid-crystal solvents provide a wealth of information, such systems do not allow for studying intermolecular relaxation in bulk hydrocarbons, which as we show in this report vary in strength across the chain and even dominates over intramolecular relaxation in the case of benzene and cyclohexane.

As a continuation of the work in Ref. 1, in this report, we also study the influence of internal motions and molecular geometry on the molecular dynamics of hydrocarbons by simulating the autocorrelation functions $G_{R, T}(t)$ of rigid $n$-alkanes (i.e., without internal motions), compared with flexible $n$-alkanes (i.e., with internal motions). While such rigid $n$-alkanes do not exist in nature, they present an ideal testing ground for simulating the influence of internal motions on correlation times and relaxation rates. We also report site-by-site simulations $G_{R, T}(t)$ for the ${ }^{1} \mathrm{H}$ 's across the chain, thereby quantifying the underlying variation in correlation times $\tau_{R, T}$ and corresponding relaxation rates $R_{1,2, R, T}$ across the molecule. The simulations of both the rigid molecules and the siteby-site ${ }^{1} \mathrm{H}$ 's reveal key insights about the functional forms of $G_{R, T}(t)$ as a function of chain length, without invoking any phenomenological models. The site-by-site simulations are also compared with total relaxation $R_{1,2}$ measurements in the case of $n$-decane and $n$-heptadecane, which show that cross-relaxation $^{40,41}$ partially (in the case of $n$-heptadecane) averages out the underlying variations in $R_{1,2}$. Such comparisons between $R_{1,2}$ measurements and site-by-site simulation could in principle be used to study cross-relaxation rates at low magnetic-fields, provided the proper theoretical framework is developed. Finally, we quantify the relative strength of intramolecular versus intermolecular relaxation as a function of internal motions, as a function of molecular geometry, and on a site-by-site basis across the chain, which to date has never been reported.

Besides addressing the fundamental science of molecular dynamics of bulk fluids, the present work also opens up new opportunities for investigating the effects of nanometer confinement on fluids, such as the liquid-state and gas-state hydrocarbons confined in the organic-matter pores of the kerogen and bitumen typically found in organic-rich shale. ${ }^{42,43}$ There is increasing evidence that the NMR surface relaxation of these light (i.e., low-viscosity) hydrocarbons confined in such organic nano-pores is dominated by intramolecular ${ }^{44}$ and intermolecular ${ }^{45}{ }^{1} \mathrm{H}-{ }^{1} \mathrm{H}$ dipole-dipole interactions, as opposed to surface paramagnetism. Provided the molecular dynamics of the bulk fluid is well understood, the MD simulations of ${ }^{1} \mathrm{H}-{ }^{1} \mathrm{H}$ dipole-dipole interactions can then in principle be used to characterize the complex NMR response of fluids confined in organic nano-pores, ${ }^{44-64}$ which as of yet is not well understood. The effects of nano-confinement on fluids in organic shale can also affect the phase behavior and partitioning of components between the matrix and production fractures as a result of the strong interaction between the fluid molecules and pore surface. It is clear that the fundamental understanding of such complex systems would significantly improve by integrating NMR measurements, MD simulations, and molecular DFT (density functional theory) ${ }^{65}$ techniques.

The rest of the article is organized as follows. Section II presents the methodology including the hydrocarbons investigated in Sec. II A, the MD simulation background in Sec. II B, and the NMR background in Sec. II C. Section III presents the results and discussions including the autocorrelation functions in Sec. III A, the rigid versus flexible molecules in Sec. III B, the site-by-site simulations in Sec III C, and crossrelaxation effects in Sec. III D. Conclusions are presented in Sec. IV.

\section{METHODOLOGY}

\section{A. Hydrocarbons investigated}

The hydrocarbons simulated in this report are shown in Fig. 1. Neopentane $\left(\mathrm{C}_{5} \mathrm{H}_{12}\right)$ refers to the isomer 2,2dimethylpropane; it is spherically symmetric, and all ${ }^{1} \mathrm{H}$ 's are equivalent. Benzene $\left(\mathrm{C}_{6} \mathrm{H}_{6}\right)$ is planar symmetric, and all ${ }^{1} \mathrm{H}$ 's are equivalent. Cyclohexane $\left(\mathrm{C}_{6} \mathrm{H}_{12}\right)$ contains two inequivalent ${ }^{1} \mathrm{H}$ 's sites, namely, axial and equatorial. Isooctane $\left(\mathrm{C}_{8} \mathrm{H}_{18}\right)$ refers to the isomer 2,2,4-trimethylpentane; it is more spherical than the normal isomer $n$-octane, with many inequivalent ${ }^{1} \mathrm{H}$ 's. The other $n$-alkanes (not shown) are labeled $n \mathrm{C \#}$ for carbon number $\mathrm{C \#}$.

The site-by-site simulations are conducted on $n$-decane $\left(n-\mathrm{C}_{10} \mathrm{H}_{22}\right)$ and $n$-heptadecane $\left(n-\mathrm{C}_{17} \mathrm{H}_{36}\right)$ labeled. In the case of $n$-decane, sites \#2 $\leftrightarrow$ \#6 have a degeneracy of 4 , while \#1 has a degeneracy of 2 . In the case of $n$-heptadecane, sites $\# 2 \leftrightarrow \# 9$ have a degeneracy of 4 , while \#1 and \#10 have a degeneracy of 2 . These degeneracies are used to compute the weighted average of the autocorrelation functions. The siteby-site simulations are also conducted on rigid $n$-decane, with the same labels as flexible $n$-decane. Note that in the case of 

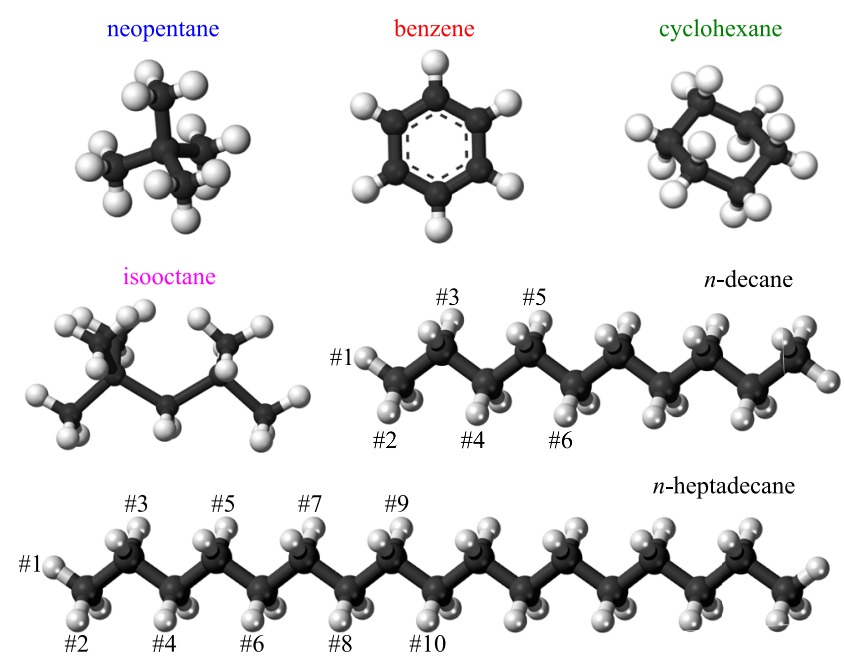

FIG. 1. Ball-and-stick illustrations of the hydrocarbons simulated in this report, including (in order of increasing molar mass) (1) neopentane $\mathrm{C}_{5} \mathrm{H}_{12}$ (2,2-dimethylpropane), (2) benzene $\mathrm{C}_{6} \mathrm{H}_{6}$, (3) cyclohexane $\mathrm{C}_{6} \mathrm{H}_{12}$, (4) isooctane $\mathrm{C}_{8} \mathrm{H}_{18}$ (2,2,4-trimethylpentane), (5) n-decane $n-\mathrm{C}_{10} \mathrm{H}_{22}$, and (6) $n$-heptadecane $n-\mathrm{C}_{17} \mathrm{H}_{36}$. $n$-decane and $n$-heptadecane also show labels for the inequivalent ${ }^{1} \mathrm{H}$ 's across the chain. The other $n$-alkanes (not shown) are labeled $n \mathrm{C} \#$ for carbon number $\mathrm{C \#}$.

flexible molecules, the distinction between sites \#1 and \#2 is not necessary, given the fast rotation of the methyl group around the $\mathrm{C}-\mathrm{C}$ axis. However, in the case of rigid molecules, the distinction between sites $\# 1$ and $\# 2$ is required and is therefore kept throughout.

\section{B. Molecular dynamics simulation}

The MD simulations of all the flexible hydrocarbons were performed using NAMD ${ }^{66}$ version 2.11 . The bulk alkanes were modeled using the CHARMM General Force field (CGenFF). ${ }^{67}$ The protocol for setting-up the initial simulation configuration was exactly as before. ${ }^{1}$ We use the $n$-alkane simulation trajectory from the earlier work for the analysis noted below. For the cyclic alkanes and isooctane, as before, we created the initial simulation system by packing $N$ copies of the molecule into a cube of volume $L^{3}$ using the Packmol program. ${ }^{68}$ The volume was chosen such that the number density $N / V$ corresponds to the experimentally determined number density at $293.15 \mathrm{~K}$. The simulation approach for these systems using NAMD was as before. ${ }^{1}$

All the rigid body simulations are performed within LAMMPS (Large-scale Atomic/Molecular Massively Parallel Simulator). ${ }^{69}$ For the rigid $n$-alkanes, we took the initial configuration from our earlier work, i.e., the configuration obtained after the Packmol packing procedure. At this stage, by construction, all the $n$-alkanes are in the fully extended (alltrans) configuration. Then using the CHARMM-to-LAMMPS tool from the Enhanced Monte Carlo package, ${ }^{70}$ we prepared the molecular configuration and force field information into a LAMMPS input data file. To remove potential steric overlaps from the packing procedure, within LAMMPS, we first run 30 steps of constant energy molecular dynamics using the NVE/LIMIT 0.1 option. (The Limit option enforces the maximum distance a particle can move and allows the simulation to proceed despite possible overlaps.) Initial velocities for the MD simulation are obtained with a Gaussian distribution and are adjusted to give a temperature of $293.15 \mathrm{~K}$. We find that 30 steps are more than sufficient to remove any potential steric overlaps and also avoid distorting the geometry of the alkanes from their original all-trans configuration.

After this dynamics step, we run rigid body molecular dynamics simulations using the RIGID/NVE/SMALL molecule option within LAMMPS. The system is thermostated using a Langevin thermostat with a damping coefficient of $5 \mathrm{ps}^{-1}$. The cutoff within LAMMPS was exactly as it was in our earlier NAMD runs for the flexible molecules. Specifically, the Lennard-Jones interactions were terminated at $14.00 \AA$ by smoothly switching to zero starting at $13.00 \AA$. The time step for integrating the equations of motion is $1 \mathrm{fs}$, and the equilibration phase was over $1 \mathrm{~ns}$. In the subsequent production phase, we remove the thermostat. The production phase lasted at least $1 \mathrm{~ns}$, and configurations are saved every 100 steps to obtain at least $10^{4}$ frames for analysis. As before, ${ }^{1}$ the average temperature in the rigid body simulations is within $\pm 3 \mathrm{~K}$ of the target temperature of $293.15 \mathrm{~K}\left(20^{\circ} \mathrm{C}\right)$. Uncertainty analysis of the autocorrelation function can be found in the supplementary material.

\section{Nuclear magnetic resonance}

Details of the derivation of the intramolecular $G_{R}(t)$ and intermolecular $G_{T}(t)^{1} \mathrm{H}-{ }^{1} \mathrm{H}$ dipole-dipole interactions and subsequent derivation of $R_{1,2}$ relaxation rates for liquids $^{5,6,8,9,71,72}$ can be found in Ref. 1 and in the supplementary material. The essential elements include the correlation times $\tau_{R, T}{ }^{8}$

$$
\tau_{R, T}=\frac{1}{G_{R, T}(0)} \int_{0}^{\infty} G_{R, T}(t) d t .
$$

In other words, $\tau_{R, T}$ is defined as the normalized area under the autocorrelation functions $G_{R, T}(t)$. The "second-moment" $\Delta \omega_{R, T}^{2}$ (i.e., the strength of the interaction) is given by the following expression: ${ }^{8}$

$$
G_{R, T}(0)=\frac{1}{3} \Delta \omega_{R, T}^{2}
$$

The intramolecular $(R)$ and intermolecular $(T)$ relaxation rates are then determined as such,

$$
R_{1,2, R, T}=\frac{1}{T_{1,2, R, T}}=\frac{10}{3} \Delta \omega_{R, T}^{2} \tau_{R, T}
$$

where $T_{1,2, R, T}$ are the corresponding relaxation times. $R_{1, R}=R_{2, R}$ and $R_{1, T}=R_{2, T}$, which is a consequence of the fast-motion regime for the liquid hydrocarbons in question. The total relaxation rates are then equal to the sum of rates

$$
R_{1,2}=\frac{1}{T_{1,2}}=\frac{10}{3} \Delta \omega_{R}^{2} \tau_{R}+\frac{10}{3} \Delta \omega_{T}^{2} \tau_{T},
$$

which is the final expression used to predict the NMR relaxation rate $R_{1}=R_{2}$ from simulation results.

In the case of $n$-decane and $n$-heptadecane, the MD simulations were compared with ${ }^{1} \mathrm{H} R_{2}$ measurements. The $n$-decane ( $\geq 99 \%$ purity) and $n$-heptadecane (99\% purity) were obtained from Sigma-Aldrich. The $n$-alkanes were de-oxygenated by bubbling high-purity $\mathrm{N}_{2}$ gas overnight and sealing the vial, thereby removing paramagnetic $\mathrm{O}_{2}$ in the solution. The NMR measurements were acquired 
at ambient conditions $\left(\simeq 25{ }^{\circ} \mathrm{C}\right)$ using a GeoSpec2 from Oxford Instruments in an $18 \mathrm{~mm}$ probe, at a Larmor frequency of $\omega_{0} / 2 \pi=2.3 \mathrm{MHz}$ for ${ }^{1} \mathrm{H}$ (where $\omega_{0}=\gamma B_{0}$, for magnetic-field strength $B_{0}$ and gyro-magnetic ratio $\gamma / 2 \pi$ $=42.57 \mathrm{MHz} / \mathrm{T}$ for $\left.{ }^{1} \mathrm{H}\right) . R_{2}$ was measured using CPMG (CarrPurcell-Meiboom-Gill) echo train with an echo spacing of $T_{E}$ $=0.2 \mathrm{~ms}$, while $R_{1}$ was measured using an inversion recovery sequence, signal averaged up to a signal-to-noise ratio of $\simeq 10^{3}$.

The measured $R_{2}$ distributions $P\left(R_{2}\right)$ were determined by performing an inversion of the following Laplace transform: ${ }^{73,74}$

$$
M(t)=\int P\left(R_{2}\right) \exp \left(-R_{2} t\right) d R_{2},
$$

where $M(t)$ is the measured magnetization decay. Due to the presence of experimental noise, the inverse Laplace transform is an ill-posed and non-unique problem, and a regularization parameter of $\simeq 10^{-3}$ is used to smooth the inverted $R_{2}$ distributions. A discrete version of Eq. (5) is used for the inversion, with evenly spaced $R_{2}$ bins, with $R_{2}>0.01 \mathrm{~s}^{-1}$. The width of the $P\left(R_{2}\right)$ distribution was found to be the same at a slightly elevated temperature of $30{ }^{\circ} \mathrm{C}$, which for the case of $n$-heptadecane rules out any potential influence from the nearby phase-transition at $\simeq 20{ }^{\circ} \mathrm{C}$. Results for $P\left(R_{1}\right)$ (not shown) were similar to $P\left(R_{2}\right)$. Note that the only quantities determined from measurements in this report are the inverted $P\left(R_{2}\right)$ distributions given in Figs. 6(b) and 7(b).

\section{RESULTS AND DISCUSSIONS}

\section{A. Autocorrelation functions}

The models developed by BPP (Bloembergen, Purcell, Pound) ${ }^{5}$ for intramolecular relaxation and Torrey ${ }^{6}$ ("TOR") for intermolecular relaxation have been the building blocks for the interpretation of NMR relaxation in liquids. Improvements to the TOR model followed with the analytic solution by Hwang and Freed ("HF") which takes into account finite boundary effects due to the distance of closest approach between spheres. More realistic pair-correlation functions for intermolecular interactions have also been studied, which introduce pair-correlation effects into the dynamical time evolution of the translational diffusion, ${ }^{7,75}$ as well as spin-eccentricity and rotation-translation coupling effects. ${ }^{76}$ Introducing such paircorrelation effects into the intermolecular relaxation model is beyond the scope of this work and will be reported elsewhere.

The BPP $G_{R}(t),{ }^{5} \mathrm{HF} G_{T}(t),{ }^{7}$ and TOR $G_{T}^{\prime}(t)^{6}$ autocorrelation functions are given by

$$
\begin{aligned}
& \frac{G_{R}(t)}{G_{R}(0)}=\exp \left(-\frac{t}{\tau_{R}}\right), \\
& \frac{G_{T}(t)}{G_{T}(0)}=\int_{0}^{\infty} \frac{54}{\pi} \frac{x^{2}}{81+9 x^{2}-2 x^{4}+x^{6}} \exp \left(-x^{2} \frac{t}{\frac{9}{4} \tau_{T}}\right) d x, \\
& \frac{G_{T}^{\prime}(t)}{G_{T}(0)}=\int_{0}^{\infty} 3 \frac{J_{3 / 2}^{2}(x)}{x} \exp \left(-x^{2} \frac{t}{\frac{5}{2} \tau_{T}}\right) d x,
\end{aligned}
$$
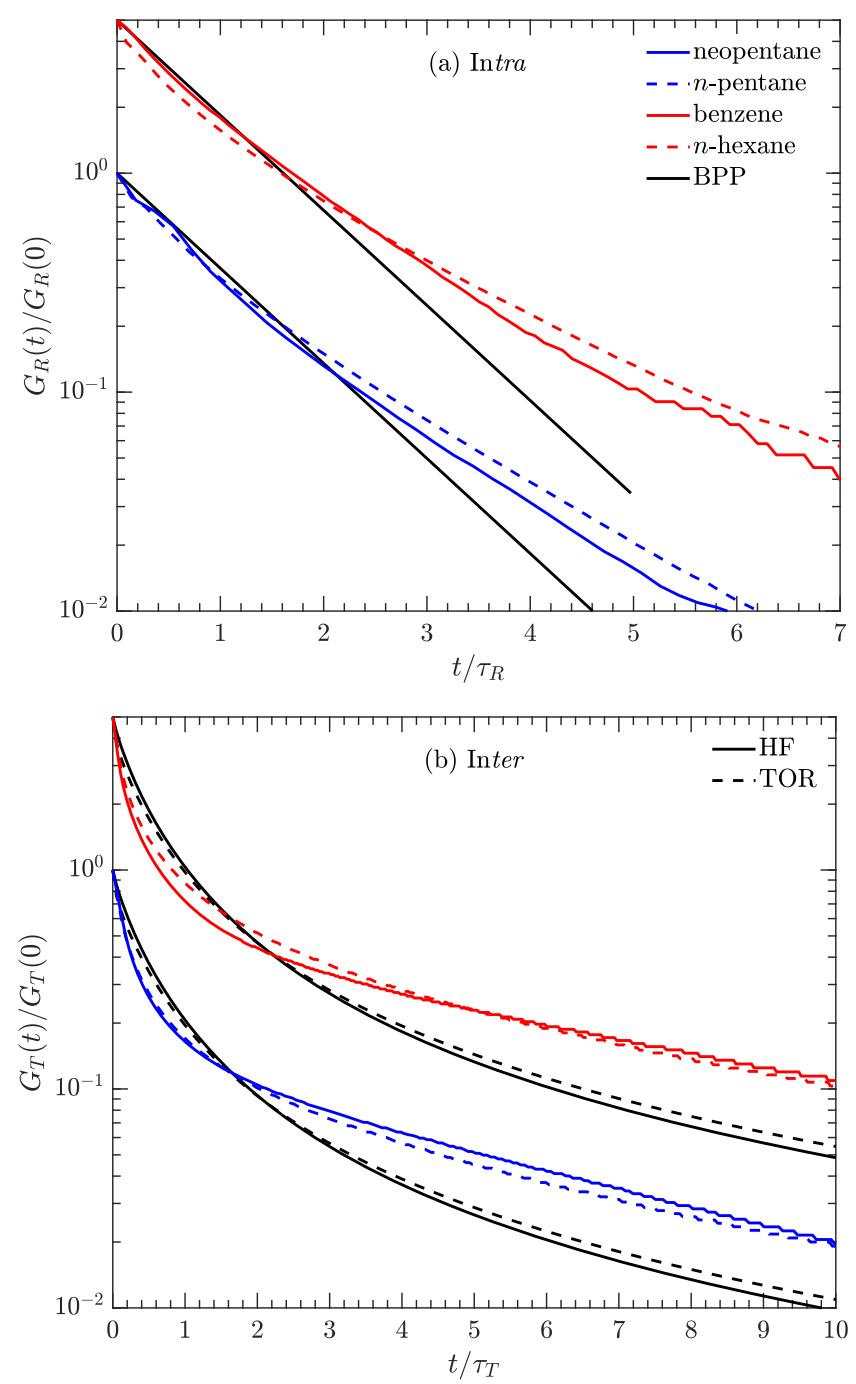

FIG. 2. MD simulations of the autocorrelation function (a) $G_{R}(t)$ for intramolecular interactions and (b) $G_{T}(t)$ for intermolecular interactions, for selected hydrocarbons defined in Fig. 1. The $y$-axis has been normalized by zero time value $G_{R, T}(0)$, and the $x$-axis has been normalized by correlation

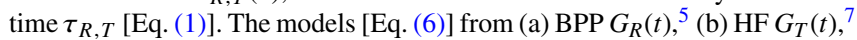
and (c) TOR $G_{T}^{\prime}(t)^{6}$ are also shown. For clarity, one set of curves has been (arbitrarily) shifted by a factor $\times 5$ along the $y$-axis.

where all expressions reduce to unity at $t=0$. The BPP model in Fig. 2(a) is a single-exponential decay [Eq. (6)], which is a straight-line decay on the semilog-y plot. The intramolecular $G_{R}(t)$ appear to be more stretched (i.e., multi-exponential like) in nature than the model; however, neopentane is clearly closer to the model than the linear isomer $n$-pentane. Similarly, benzene is closer to the BPP model, relative to $n$-hexane. A more quantitative analysis of $G_{R}(t)$ is detailed in the supplementary material, which decomposes $G_{R, T}(t)$ into a distribution in correlation times using inverse Laplace transforms, solely for the purpose of comparing with the BPP and HF models.

Meanwhile, the HF model for the intermolecular autocorrelation $G_{T}(t)$ is plotted in Fig. 2(b). The data indicate that molecular symmetry does not have a significant effect on the functional form of $G_{T}(t)$ and remains equally departed from the model. The TOR model $G_{T}^{\prime}(t)$ is similar to HF, although the TOR model is slightly more stretched than HF. 
Another way to quantify deviations from the models is to compute the ratio of the translational-diffusion correlation time $\tau_{D}$ to the rotational-diffusion correlation time $\tau_{R}$, which according to the Stokes-Einstein relation should be $\tau_{D} / \tau_{R}$ $=9$ for spherical molecules. ${ }^{9}$ Note that in order to relate the Stokes-Einstein translational-diffusion correlation time $\tau_{D}$ to the NMR derived translational correlation time $\tau_{T}$ [Eq. (1)], a factor of $\frac{9}{4}$ is required, i.e., $\tau_{D}=\frac{9}{4} \tau_{T}^{8}$ according to the HF model. In the case of neopentane, the ratio is found to be $\tau_{D} / \tau_{R}$ $=7.88$, which is consistent with the prediction. More details about the BPP, HF, and Stokes-Einstein relation can be found in the supplementary material.

\section{B. Rigid versus flexible molecules}

As discussed above, neopentane is a spherical molecule with relatively stiff (i.e., rigid) bonds and is therefore expected to be consistent with the Stokes-Einstein relation of spherical molecules. We now turn our attention to the effects of internal motions in the long-chain $n$-alkanes, which we study here by simulating completely rigid $n$-alkanes. While rigid molecules do not exist in nature, they provide an ideal testing ground for quantifying the effects of rigidity on the molecular dynamics and the NMR relaxation. The characterization of internal motions in hydrocarbons originates from Woessner's theories, ${ }^{13}$ who postulated that internal motions would shorten the correlation times $\tau_{R, T}$ and therefore reduce the relaxation rates $R_{1,2}$ in liquids, which we confirm below. Furthermore, it was postulated in Ref. 13 that internal motions would cause the intramolecular contribution to relaxation $\left(R_{1,2, R}\right)$ to decrease relative to the intermolecular contribution $\left(R_{1,2, T}\right)$, which we also confirm below. Put in other words, we can test the original theories using MD simulations, without invoking a phenomenological model.

The effects of internal motions on intramolecular autocorrelation functions $G_{R}(t)$ for $n$-pentane $\leftrightarrow n$-decane are shown in Fig. 3, presented in such a way as to allow for direct comparison between (a) rigid molecules and (b) flexible molecules. The internal motions clearly decrease the correlation times $\tau_{R}$, as shown when going from (a) rigid to (b) flexible molecules. The corresponding intermolecular autocorrelation functions $G_{T}(t)$ show similar trends (see the supplementary material). What is also significant is that the stretched decay for intramolecular $G_{R}(t)$ in Fig. 3 persists for rigid molecules, indicating that molecular geometry plays a crucial role in the functional form of $G_{R}(t)$. If internal motions were the only cause of the stretched decay, then the functional forms for rigid $G_{R}(t)$ (which do not have internal motions) would be straighter (i.e., closer to single exponential). In fact, analysis of the $G_{R}(t)$ decay indicates that internal motions have a tendency to narrow the underlying distribution in correlation times, i.e., make $G_{R}(t)$ closer to single exponential decay, for $n$-nonane and above (see the supplementary material).

The correlation times $\tau_{R, T}$ computed using Eq. (1) for rigid and flexible molecules are plotted in Fig. 4 for (a) intramolecular and (b) intermolecular interactions. The rotational correlation-time $\tau_{R}$ for flexible benzene agrees well with previous estimates from NMR measurements and MD simulations. ${ }^{15,16}$ In the case of rigid molecules, the maximum autocorrelation time $t=150 \mathrm{ps}$ did not fully capture
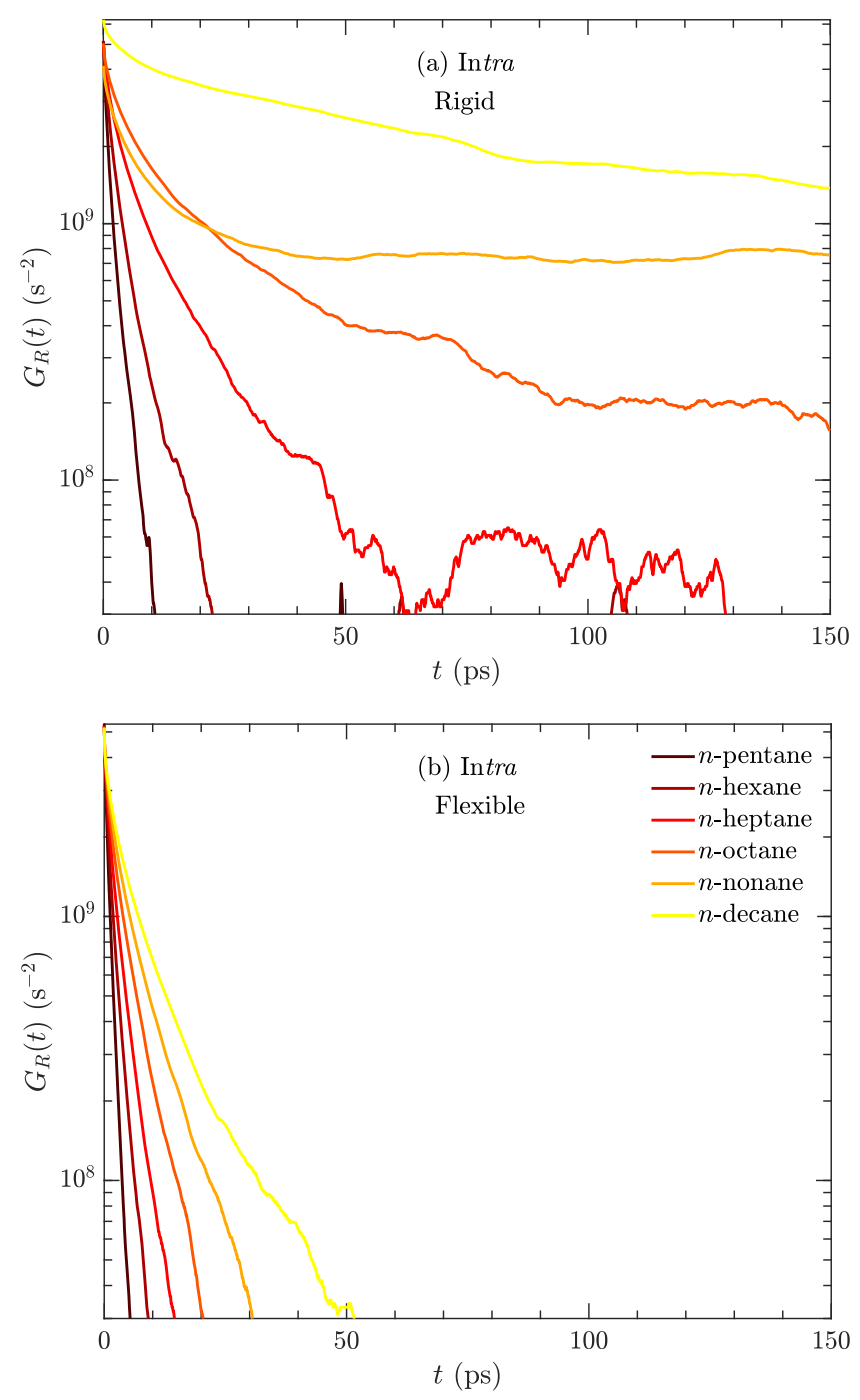

FIG. 3. MD simulations of the autocorrelation function $G_{R}(t)$ for intramolecular interactions, for (a) rigid and (b) flexible hydrocarbons, from $n$-pentane to $n$-decane.

the decay in $G_{R, T}(t)$, implying that $\tau_{R, T}$ is underestimated for rigid $n$-nonane and rigid $n$-decane, and therefore $R_{1,2}$ is also underestimated. Nevertheless, Fig. 4(a) shows that the estimated intramolecular $\tau_{R}$ is a factor $\simeq 2$ greater for rigid $n$-pentane than flexible $n$-pentane, and a factor $\simeq 12$ greater for rigid $n$-decane than flexible $n$-decane. Meanwhile, Fig. 4(b) shows that the estimated intermolecular $\tau_{T}$ is roughly the same between rigid $n$-pentane and flexible $n$-pentane but a factor $\simeq 5$ greater for rigid $n$-decane than flexible $n$-decane. These findings clearly imply that internal motion effects become more prominent with increasing chain-length.

The next step is to compute the intramolecular $R_{1,2, R}$ and intermolecular $R_{1,2, T}$ relaxation rates from Eq. (3). Figure 5(a) shows the ratio in relaxation rates $R_{1,2, R} / R_{1,2, T}$, where $R_{1,2, R} / R_{1,2, T} \gg 1$ indicates that intramolecular relaxation dominates over intermolecular relaxation, while $R_{1,2, R} / R_{1,2, T} \ll 1$ indicates that intermolecular relaxation dominates over intramolecular relaxation instead. The data indicate that internal motions decrease the intramolecular contribution relative to the intermolecular contribution by a factor $\simeq 2$ on average, although some scattering exist in the $R_{1,2, R} / R_{1,2, T}$ data 

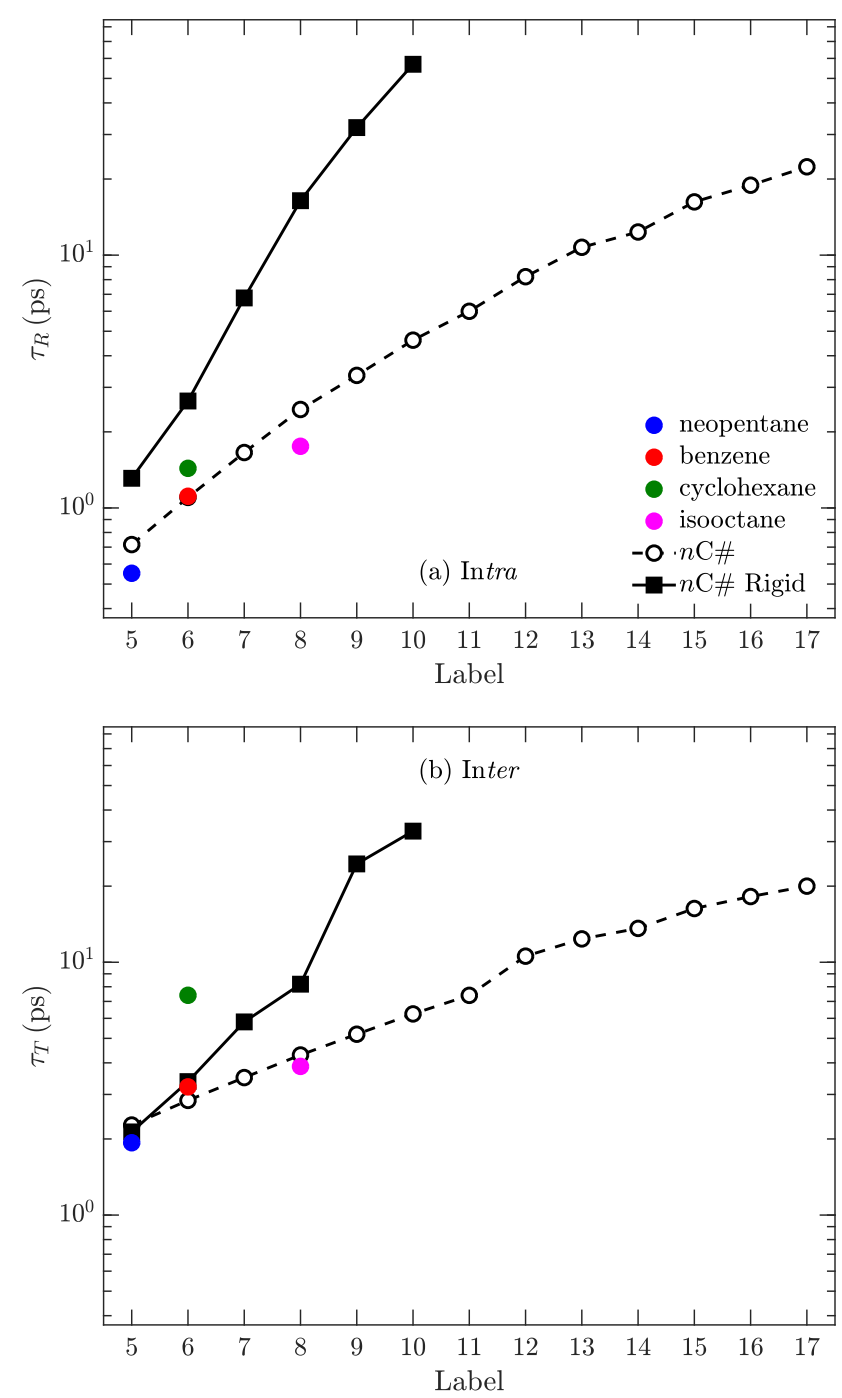

FIG. 4. MD simulation results for correlation times from (a) intramolecular $\tau_{R}$ and (b) intermolecular $\tau_{T}$ interactions, using Eq. (1). Hydrocarbons are defined in Fig. 1 and labeled in the order of increasing carbon number $\mathrm{C \#}$. "Rigid" refers to rigid hydrocarbons, while all others are flexible. Lines are a guide for the eye.

for rigid $n$-nonane and rigid $n$-decane. This is consistent with the predictions in Ref. 13. These results can also be analyzed in terms of the ratio $\tau_{D} / \tau_{R}$ (where $\tau_{D}=\frac{9}{4} \tau_{T}$ ), which shows that $\tau_{D} / \tau_{R}$ for rigid molecules is on average by a factor $\simeq 2$ further away from the spherical model (see the supplementary material).

Finally, Fig. 5(b) presents the total relaxation rate $R_{1,2}$ computed using Eq. (4), showing that $R_{1,2}$ is a factor $\simeq 1.4$ larger for rigid $n$-pentane than flexible $n$-pentane, while it is a factor $\simeq 13$ larger for rigid $n$-decane than flexible $n$-decane. Again, these findings clearly imply that internal motion effects become more prominent with increasing chain-length, in line with predictions from Ref. 13.

For the flexible isomers discussed in Sec. III A, Fig. 5 shows that while neopentane and benzene show results consistent with their corresponding $n$-alkane, benzene and cyclohexane clearly do not. More specifically, Fig. 5(a) shows that for all cases, relaxation is dominated by intramolecular interactions, i.e., $R_{1,2, R} / R_{1,2, T} \gg 1$, except for benzene and cyclohexane
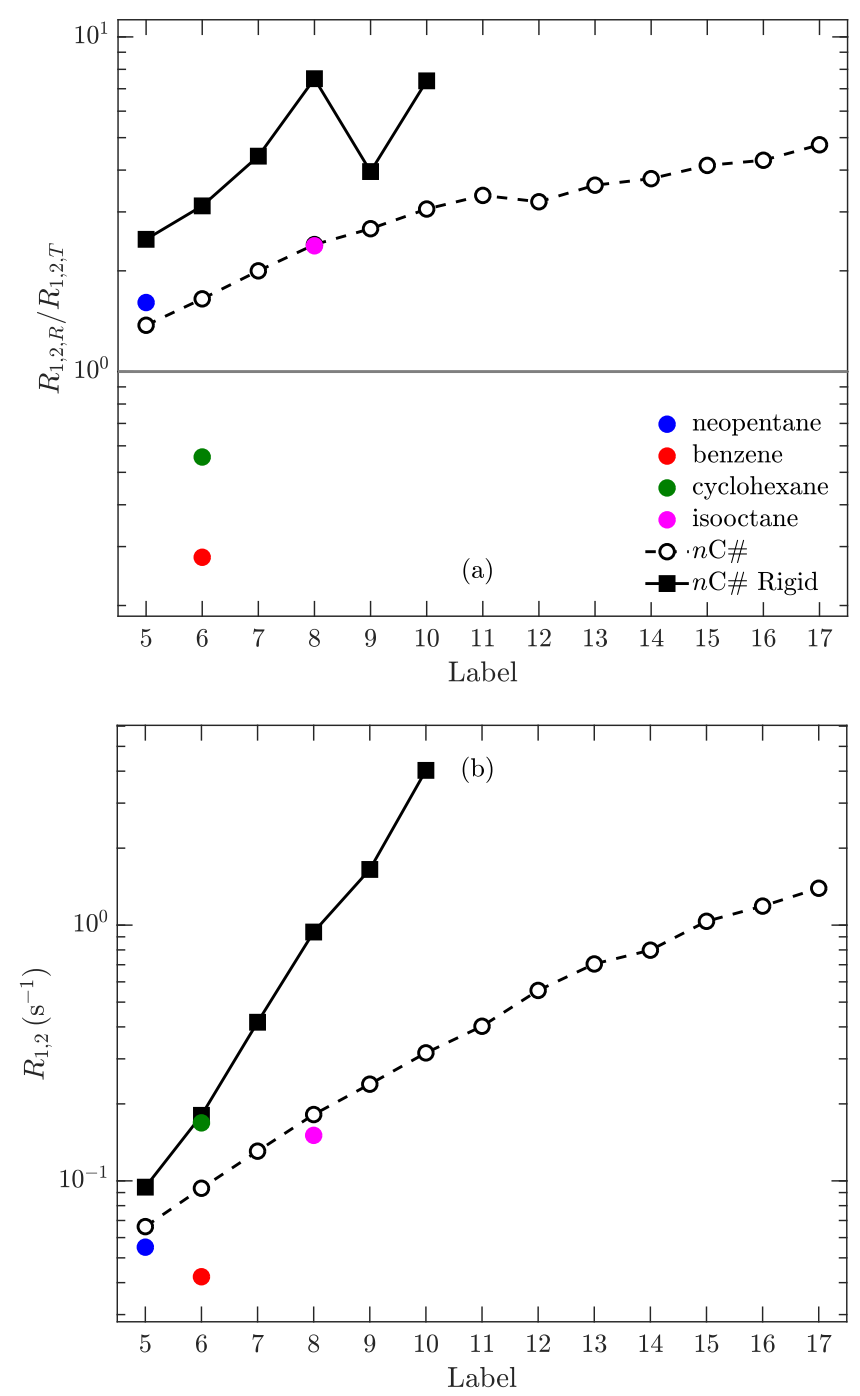

FIG. 5. MD simulation results for (a) the ratio of relaxation rates $R_{1,2, R} / R_{1,2, T}$ using Eq. (3) and (b) the total relaxation rate $R_{1,2}$, using Eq. (4). Hydrocarbons are defined in Fig. 1 and labeled in the order of increasing carbon number C\#. "Rigid" refers to rigid hydrocarbons, while all others are flexible. Lines are a guide for the eye.

which show that $R_{1,2}$ is dominated by intermolecular interactions instead, i.e., $R_{1,2, R} / R_{1,2, T} \ll 1$. In the case of benzene, this is a result of a lower second-moment $\Delta \omega_{R}^{2}$ (see the supplementary material) compared to all the other $n$-alkanes. In the case of cyclohexane, this is a result of a larger $\tau_{T}$ [Fig. 4(b)] compared to $n$-hexane. These differences result in the spread of $R_{1,2}$ [Fig. 5(b)] for benzene and cyclohexane compared with $n$-hexane.

\section{Site-by-site simulations}

The above results show that internal motions are not the only cause of the functional form of $G_{R, T}(t)$. Our next task is therefore to determine whether the stretched decays in $G_{R, T}(t)$ are a result of underlying variations in molecular dynamics across the chain-length. This scenario was previously postulated, ${ }^{41}$ where the fast rotation (i.e., short $\tau_{R}$ ) of the methyl groups acts as relaxation sinks for the macro-molecule.

In order to investigate variations across the chain, we perform the MD simulations for each ${ }^{1} \mathrm{H}$ across the chain and 
then compute the correlation times $\tau_{R, T}$ and relaxation rates $R_{1,2}$ on a site-by-site basis across the chain. The ${ }^{1} \mathrm{H}$ sites are labeled in Fig. 1 for $n$-decane and $n$-heptadecane, where \#1, $\# 2{ }^{1} \mathrm{H}$ 's are on the methyl end-group, and the largest number is in the middle of the chain (more details in Sec. II A). Intramolecular $G_{R}(t)$ for $n$-heptadecane in Fig. 6(a) shows a large variation in correlation times, with the $\# 1, \# 2^{1} \mathrm{H}$ 's on the methyl clearly having the steepest decay (i.e., shortest $\tau_{R}$ ), as expected from Ref. 41. The average "Ave" is the weighted average over all sites and constitutes what was reported in Secs. III A and III B. Meanwhile, intermolecular $G_{T}(t)$ for $n$-heptadecane (see the supplementary material) shows less variation between sites than intramolecular, which is intuitive since the distance of closest approach between molecules should be roughly independent of the location across the chain. The same site-by-site method is used for $n$-decane in Fig. 7(a), where the intramolecular $G_{R}(t)$ and the intermolecular $G_{T}(t)$
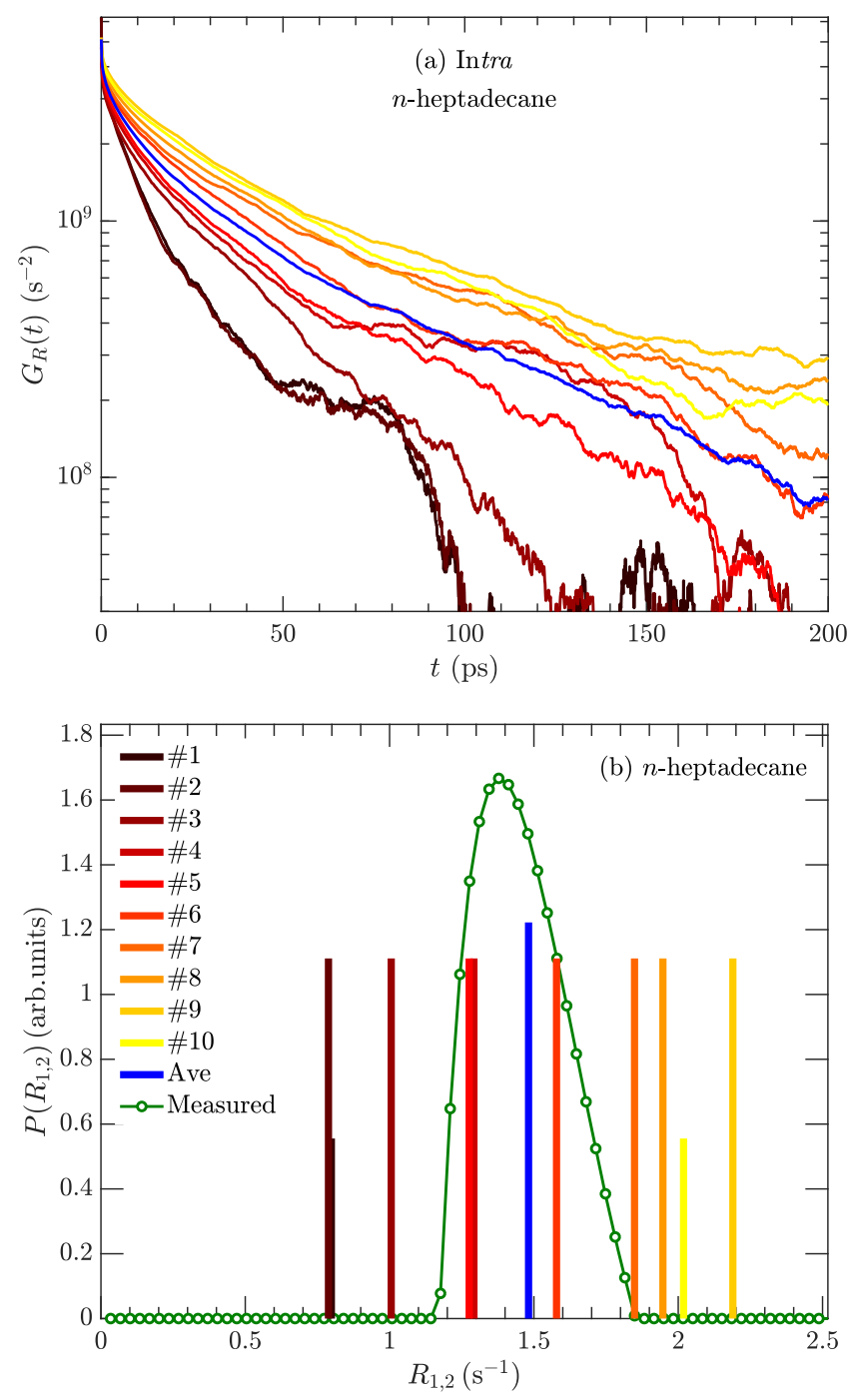

FIG. 6. MD simulation results of (a) the autocorrelation function for intramolecular $G_{R}(t)$, for $n$-heptadecane on a site-by-site basis with labels defined in Fig. 1, where "Ave" indicates weighted average over the siteby-site results, and (b) the variation in site-by-site values of $R_{1,2, i}$ (i.e., total relaxation rate) for $n$-heptadecane, with relative heights according to degeneracy, and "Ave" of arbitrary height. Also shown is the measured distribution in $R_{2}$ (Sec. II C), where the line between data points is a guide for the eye.
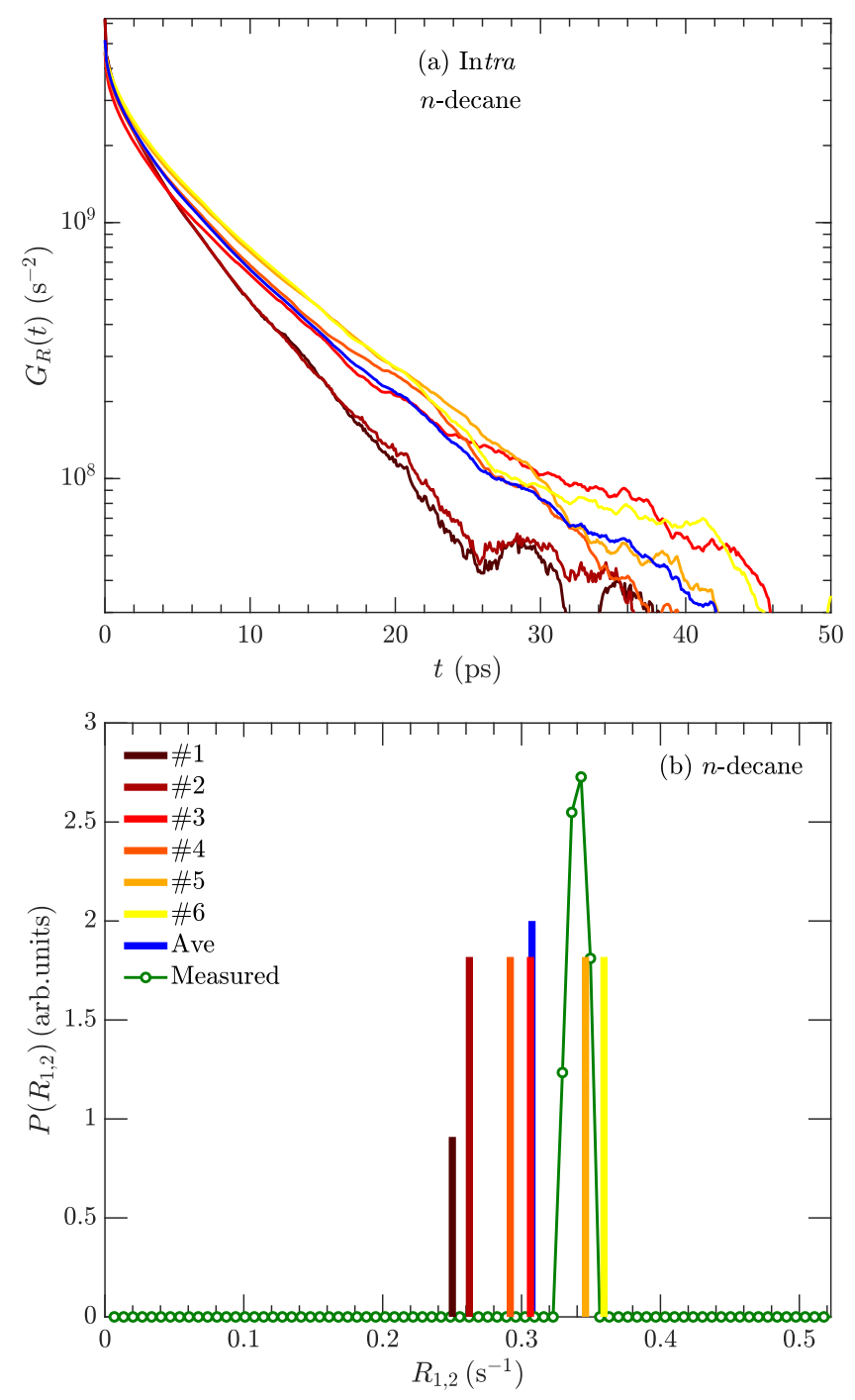

FIG. 7. The same as Fig. 6, but for $n$-decane.

(see the supplementary material) show less variation between sites than $n$-heptadecane. This is intuitive since one expects there to be more variation in the molecular dynamics across longer chains.

A more quantitative summary of the site-by-site correlation times $\tau_{R, T}$ [using Eq. (1)] is given in Fig. 8, for both $n$-decane and $n$-heptadecane. In the case of $n$-heptadecane, the ${ }^{1} \mathrm{H}$ 's labeled \#1, \#2 (both on the methyl end-group) show a factor $\simeq 4$ shorter intramolecular $\tau_{R}$ than at the middle of the chain. In the case of $n$-decane, $\# 1, \# 2$ show a factor $\simeq 2$ shorter intramolecular $\tau_{R}$ than at the middle of the chain. Also shown in Fig. 8 are results from the site-by-site simulation for rigid $n$-decane. It is interesting to note that for rigid $n$-decane, $\# 1$, $\# 2$ only show a factor $\simeq 1.4$ shorter intramolecular $\tau_{R}$ than the chain middle, which indicates less variation than for flexible $n$ decane. In other words, the internal motions have a tendency to enhance the site-by-site variation of intramolecular $\tau_{R}$ across the chain.

In the case of intermolecular $\tau_{T}$ (see the supplementary material), both rigid and flexible $n$-decane (as well as flexible $n$-heptadecane) show the same factor $\simeq 1.4$ shorter $\tau_{T}$ for \#1, $\# 2$ versus the chain middle, which is reasonable given that the 

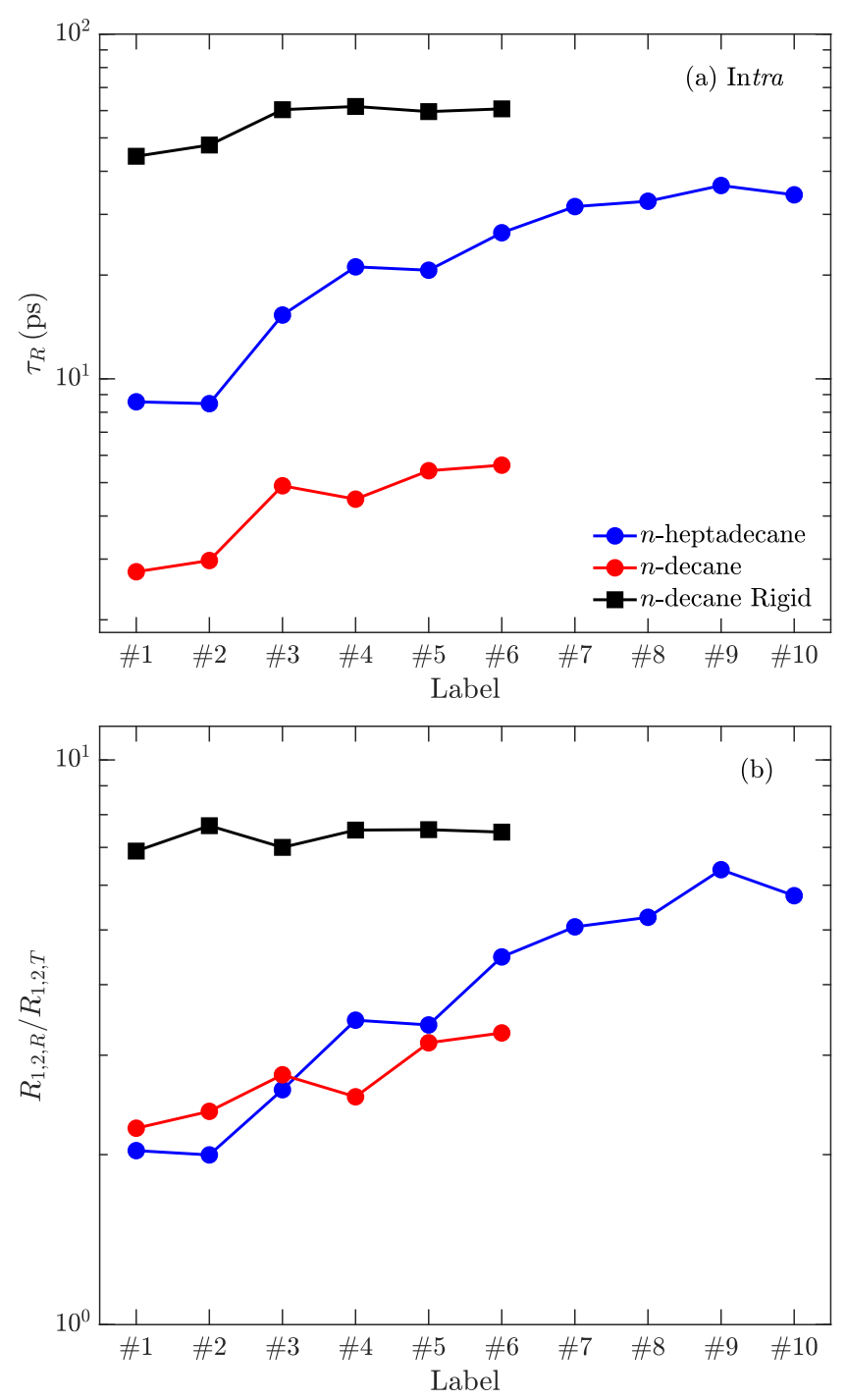

FIG. 8. MD simulation results for (a) correlation times from intramolecular $\tau_{R}$ interactions, using Eq. (1), on a site-by-site basis with site labels defined in Fig. 1 and (b) the ratio of relaxation rates $R_{1,2, R} / R_{1,2, T}$ using Eq. (3). Hydrocarbons are defined in Fig. 1 and labeled in the order of position into the chain. "Rigid" refers to rigid hydrocarbons, while all others are flexible. Lines are a guide for the eye.

distance of closest approach between molecules should be the same across the chain, regardless of whether the molecule is rigid or not.

Figure 8(b) presents the ratio $R_{1,2, R} / R_{1,2, T}$ across the chain sites, which shows that the intramolecular relaxation dominates in the chain middle (i.e., $R_{1,2, R} / R_{1,2, T} \gg 1$ ), while intramolecular relaxation is a factor of $\simeq 2$ stronger than intermolecular at the chain ends (i.e., \#1, \#2). In the case of $n$-heptadecane, the chain middle shows a factor $\simeq 3$ larger value of $R_{1,2, R} / R_{1,2, T}$ than the chain ends. In the case of $n$-decane, the chain middle shows only a factor $\simeq 1.5$ larger value of $R_{1,2, R} / R_{1,2, T}$ than the chain ends, which is reasonable given the smaller variation in correlation times $\tau_{R, T}$ for $n$-decane. Rigid $n$-decane shows almost no variation in $R_{1,2, R} / R_{1,2, T}$ $\simeq 7$, and clearly intramolecular relaxation dominates across the entire chain.

Given the large variation in $\tau_{R}$ across the chain (Fig. 8), one expects the average $G_{R}(t)$ to be a multi-exponential (i.e., stretched) decay, since the average $G_{R}(t)$ is the weighted sum of decays over all sites. Indeed $n$-heptadecane [Fig. 6(a)] and $n$-decane [Fig. 7(a)] show a stretched decay for the average. What is remarkable, however, is that $G_{R}(t)$ for both $n$-heptadecane and $n$-decane show a stretched decay at every site. This indicates that molecular geometry and internal motions play a crucial role in the functional form in the decay of $G_{R}(t)$ at each site.

\section{Cross-relaxation effects}

Figures 6(b) and 7(b) present the total relaxation rate $R_{1,2}$ on a site-by-site basis and in the form of a distribution function $P\left(R_{1,2}\right)$. In the case of $n$-heptadecane [Fig. 6(b)], the chain middle shows a factor $\simeq 3$ larger value of $R_{1,2}$ than the chain ends. In the case of $n$-decane [Fig. 7(b)], the chain middle shows only a factor $\simeq 1.5$ larger value of $R_{1,2}$ than the chain ends, which is reasonable given the smaller variation in correlation times $\tau_{R, T}$ in Fig. 8. Despite these site-by-site variations in $R_{1,2}$ across the chain, the measured distributions have narrower widths than the site-by-site simulations would predict. This is a result of cross-relaxation effects, a.k.a. "spin diffusion" effects, which tend to average out (i.e., "wash out") any such variations across the chain. ${ }^{41,77-79}$

The condition for either strong, intermediate, or weak cross-relaxation is determined by the relative strength of the cross-relaxation rate $\sigma_{1,2, i j}$ between spin-pairs $i$ and $j$ compared with the difference in individual relaxation rates $\left|R_{1,2, i}-R_{1,2, j}\right|$ (see the supplementary material for more details). More specifically, the different cross-relaxation regimes can be defined as such, 3,41

$$
\begin{aligned}
\sigma_{1,2, i j} \gg \frac{1}{2}\left|R_{1,2, i}-R_{1,2, j}\right| \quad \text { (strong), } \\
\sigma_{1,2, i j} \simeq \frac{1}{2}\left|R_{1,2, i}-R_{1,2, j}\right| \quad \text { (intermediate), } \\
\sigma_{1,2, i j} \ll \frac{1}{2}\left|R_{1,2, i}-R_{1,2, j}\right| \quad \text { (weak). }
\end{aligned}
$$

For strong cross-relaxation, which is generally the case for low-viscosity liquids, one expects the measured $R_{1,2}$ to be single-valued and given by the average rate ${ }^{11,41}$

$$
R_{1,2}=\frac{1}{N} \sum_{i} R_{1,2, i}
$$

where $N$ is the number of ${ }^{1} \mathrm{H}$ 's in the chain, and $R_{1,2, i}$ is the relaxation rate of the $i^{\prime}$ th ${ }^{1} \mathrm{H}$ in the chain shown in Figs. 6(b) and 7 (b). One consequence of averaging the relaxation rates in Eq. (8) is that the average $R_{1,2}$ is equivalent to computing the weighted average $G_{R, T}(t)$ in Figs. 6 and 7 and then using Eqs. (1)-(4).

In the case of $n$-heptadecane in Fig. 6(b), the simulation predicts an average value of $R_{1,2} \simeq 1.48 \mathrm{~s}^{-1}$, which is close to the measured value of $R_{1,2} \simeq 1.45 \mathrm{~s}^{-1}$. In the case of $n$-decane in Fig. 7(b), the simulation predicts an average value of $R_{1,2} \simeq 0.308 \mathrm{~s}^{-1}$, which is also close to the measured value of $R_{1,2} \simeq 0.328 \mathrm{~s}^{-1}$. Such agreement was previously reported in Ref. 1, which validates the force fields used in the simulation. 
However, while the mean values agree, Figs. 6(b) and 7(b) clearly show that the widths of simulation versus measurement do not agree. In the case of $n$-heptadecane, the simulation predicts a width of $\Delta_{1,2} \simeq 1.40 \mathrm{~s}^{-1}$ (defined as the difference between maximum and minimum values in the variation), which is a factor $\simeq 3$ times larger than the measured full-width at half-maximum $W_{1,2} \simeq 0.42 \mathrm{~s}^{-1}$ of the distribution. The $n$-heptadecane results therefore indicate that crossrelaxation partially averages out the site-by-site variation in relaxation. In other words, in the case of weak cross-relaxation $\sigma_{1,2, i j} \ll \frac{1}{2}\left|R_{1,2, i}-R_{1,2, j}\right|$, the measured $W_{1,2}$ would agree with $\Delta_{1,2} \simeq 1.40 \mathrm{~s}^{-1}$ from simulation. In the case of strong cross-relaxation $\sigma_{1,2, i j} \gg \frac{1}{2}\left|R_{1,2, i}-R_{1,2, j}\right|$, the measured $W_{1,2}$ would tend toward zero [i.e., the $P\left(R_{1,2}\right)$ would tend toward a delta function], which in practice would be limited by the experimental resolution of $\simeq 0.06 R_{1,2}$ (as determined on a water sample). Figure 6(b) indicates that $\sigma_{1,2, i j}$ is somewhere inbetween the strong and weak cross-relaxation regime, i.e., in the intermediate regime, suggesting that $\sigma_{1,2, i j}$ could in principle be calculated from the observed difference between simulation and measurements at low magnetic-fields $\left(\omega_{0} / 2 \pi\right.$ $\lesssim 2.3 \mathrm{MHz}$ ).

In the case of $n$-decane [Fig. 7(b)], the simulation predicts a width of $\Delta_{1,2} \simeq 0.11 \mathrm{~s}^{-1}$, which is much larger than the measured $W_{1,2} \simeq 0.02 \mathrm{~s}^{-1}$. Given that the experimental resolution limit has been reached, the true measured width for $n$-decane must satisfy $W_{1,2}<0.02 \mathrm{~s}^{-1}$. This implies that $\Delta_{1,2}$ is at least a factor $\gtrsim 6$ times larger than $W_{1,2}$, indicating that cross-relaxation is more efficient for $n$-decane than for $n$-heptadecane.

\section{CONCLUSIONS}

We show how the functional forms of the autocorrelation functions for intramolecular $G_{R}(t)$ and intermolecular $G_{T}(t)$ ${ }^{1} \mathrm{H}-{ }^{1} \mathrm{H}$ dipole-dipole interactions depend on the symmetry of the molecule. More specifically, we show that hydrocarbons with increased molecular symmetry such as neopentane and benzene show better agreement with traditional relaxation models ${ }^{5-7}$ than their corresponding straight-chain $n$-alkane, most likely a result of the increasing influence of internal motions and molecular geometry with increasing chain-length. Furthermore, we show that the spherically symmetric neopentane is consistent with the Stokes-Einstein relation for diffusion of spherical molecules.

We determine the correlation times $\tau_{R, T}$ and corresponding ${ }^{1} \mathrm{H}$ NMR relaxation rates $R_{1,2, R, T}$ for intramolecular $(R)$ and intermolecular $(T)$ interactions, respectively. By comparing the relaxation of rigid and flexible $n$-alkanes, we find a factor $\simeq 12$ increase in the rotational correlation-time $\tau_{R}$ for rigid $n$-decane compared with flexible $n$-decane, together with a factor $\simeq 5$ increase in the translational correlation-time $\tau_{T}$, thereby revealing the strong influence of internal motions on $R_{1,2, R, T}$ for long-chain $n$-alkanes. We find that relaxation from intramolecular interactions dominates over intermolecular interactions (i.e., $R_{1,2, R} / R_{1,2, T} \gg 1$ ) for all hydrocarbons investigated, except for benzene and cyclohexane where intermolecular interactions dominate (i.e., $R_{1,2, R} / R_{1,2, T} \ll 1$ ). The rigid $n$-alkanes show a factor $\simeq 2$ larger ratio $R_{1,2, R} / R_{1,2, T}$ than flexible $n$-alkanes, indicating that internal motions somewhat diminish the influence of intramolecular interactions, as predicted by Woessner. ${ }^{13}$

Site-by-site simulations of $G_{R, T}(t)$ for the ${ }^{1} \mathrm{H}$ 's across the chain indicate that $\tau_{R}$ decreases by a factor $\simeq 4$ toward the chain-ends of $n$-heptadecane, together with a factor $\simeq 1.4$ decrease in $\tau_{T}$, thereby revealing variations in $\tau_{R, T}$ and $R_{1,2, R, T}$ across the chain for long-chain $n$-alkanes. In the case of $n$-heptadecane, the chain middle shows a factor $\simeq 3$ larger value of $R_{1,2, R} / R_{1,2, T}$ than the chain ends, implying that intramolecular relaxation dominates more in the chain middle. The simulations indicate that the stretched functional-forms of siteby-site $G_{R, T}(t)$ are approximately the same as the chain averages, indicating that molecular geometry and internal motions play a crucial role in the functional form of $G_{R, T}(t)$ at each site.

Measurements of $n$-heptadecane indicate a narrower distribution in the total relaxation rate $R_{1,2}$ than site-by-site simulations, implying that cross-relaxation (partially) averages-out the variations in $R_{1,2}$ across the chain of long-chain $n$-alkanes. Such comparisons between site-by-site simulations and measurements at low magnetic-field $\left(\omega_{0} / 2 \pi \lesssim 2.3 \mathrm{MHz}\right)$ could in principle be used to compute the cross-relaxation rates $\sigma_{1,2, i j}$ for long-chain $n$-alkanes.

\section{SUPPLEMENTARY MATERIAL}

See supplementary material for (A) uncertainty analysis, (B) further background material on the NMR formalism, (C) greater discussion on the Stokes-Einstein relation, (D) decomposition of $G_{R, T}(t)$ into a distribution in correlation times using inverse Laplace transforms, (E) further results on rigid versus flexible molecules, (F) further results on the site-by-site simulations, $(\mathrm{G})$ a discussion on cross-relaxation, and $(\mathrm{H})$ tables collecting all the data.

\section{ACKNOWLEDGMENTS}

This work was funded by the Rice University Consortium on Processes in Porous Media and the American Chemical Society Petroleum Research Fund (No. ACS-PRF58859-ND6). We gratefully acknowledge the National Energy Research Scientific Computing Center, which is supported by the Office of Science of the U.S. Department of Energy (No. DE-AC02-05CH11231), for HPC time and support. We also gratefully acknowledge the Texas Advanced Computing Center (TACC) at The University of Texas at Austin (URL: http://www.tacc.utexas.edu) for providing HPC resources and the reviewers for their helpful comments.

${ }^{1}$ P. M. Singer, D. Asthagiri, W. G. Chapman, and G. J. Hirasaki, J. Magn. Reson. 277, 15 (2017).

${ }^{2}$ D. E. Woessner, J. Chem. Phys. 41(1), 84 (1964).

${ }^{3}$ J. Kowalewski and L. Mäler, Nuclear Spin Relaxation in Liquids: Theory, Experiments, and Applications (Taylor \& Francis Group, 2006).

${ }^{4}$ P. Henritzi, A. Bormuth, and M. Vogel, Solid State Nucl. Magn. Reson. 54, 32 (2013).

${ }^{5}$ N. Bloembergen, E. M. Purcell, and R. V. Pound, Phys. Rev. 73(7), 679 (1948).

${ }^{6}$ H. C. Torrey, Phys. Rev. 92(4), 962 (1953).

${ }^{7}$ L.-P. Hwang and J. H. Freed, J. Chem. Phys. 63(9), 4017 (1975). 
${ }^{8}$ B. Cowan, Nuclear Magnetic Resonance and Relaxation (Cambridge University Press, 1997).

${ }^{9}$ A. Abragam, Principles of Nuclear Magnetism, International Series of Monographs on Physics (Oxford University Press, 1961).

${ }^{10}$ D. E. Woessner, J. Chem. Phys. 36(1), 1 (1962).

${ }^{11}$ D. E. Woessner, J. Chem. Phys. 37(3), 647 (1962).

${ }^{12}$ W. T. Huntress, J. Chem. Phys. 48(8), 3524 (1968).

${ }^{13}$ D. E. Woessner, J. Chem. Phys. 42(6), 1855 (1965).

${ }^{14}$ P. S. Hubbard, J. Chem. Phys. 51(4), 1647 (1969).

${ }^{15}$ A. Laaksonen, P. Stilbs, and R. E. Wasylishen, J. Chem. Phys. 108(2), 455 (1998).

${ }^{16}$ R. Witt, L. Sturz, A. Dölle, and F. Müller-Plathe, J. Phys. Chem. A 104, 5716 (2000).

${ }^{17}$ G. Lipari and A. Szabo, J. Am. Chem. Soc. 104, 4546 (1982).

${ }^{18}$ G. Lipari and A. Szabo, J. Am. Chem. Soc. 104, 4559 (1982).

${ }^{19}$ S. Kariyo, A. Brodin, C. Gainaru, A. Herrmann, H. Schick, V. N. Novikov, and E. A. Rössler, Macromolecules 41, 5313 (2008).

${ }^{20}$ D. Frueh, Prog. Nucl. Magn. Reson. Spectrosc. 41, 305 (2002).

${ }^{21}$ P. A. Beckmann, Phys. Rep. 171(3), 85 (1988).

${ }^{22}$ V. I. Bakhmutov, NMR Spectroscopy in Liquids and Solids (CRC Press, Taylor \& Francis Group, 2015).

${ }^{23}$ A. Bormuth, M. Hofmann, P. Henritzi, M. Vogel, and E. A. Rössler, Macromolecules 46, 7805 (2013).

${ }^{24}$ P. M. Singer, Z. Chen, L. B. Alemany, G. J. Hirasaki, K. Zhu, Z. H. Xie, and T. D. Vo, in SPWLA 58th Annual Logging Symposium, 17-21 June, Oklahoma City, Oklahoma, USA (Society of Petrophysicists and Well-Log Analysts, 2017), SPWLA-2017-XX.

${ }^{25}$ P. M. Singer, Z. Chen, L. B. Alemany, G. J. Hirasaki, K. Zhu, Z. H. Xie, and T. D. Vo, Energy Fuels 32(2), 1534 (2018).

${ }^{26}$ C. Calero, J. Martí, and E. Guàrdia, J. Phys. Chem. B 119, 1966 (2015).

${ }^{27}$ W. A. M. Madhavi, S. Weerasinghe, and K. I. Momot, J. Phys. Chem. B 121, 10893 (2017).

${ }^{28}$ E. E. Burnell and C. A. de Lange, J. Magn. Reson. 39, 461 (1980).

${ }^{29}$ J. W. Emsley and G. R. Luckhurst, Mol. Phys. 41(1), 19 (1980).

${ }^{30}$ R. R. Vold, R. L. Vold, and N. M. Szeverenyl, J. Phys. Chem. 85, 1934 (1981).

${ }^{31}$ J. W. Emsley, G. R. Luckhurst, and C. P. Stockley, Mol. Phys. 44(3), 565 (1981).

${ }^{32}$ P. A. Beckmann, J. W. Emsley, G. R. Luckhurst, and D. L. Turner, Mol. Phys. 50(4), 699 (1983).

${ }^{33}$ C. R. J. Counsell, J. W. Emsley, G. R. Luckhurst, D. L. Turner, and J. Charvolin, Mol. Phys. 52(2), 499 (1984).

${ }^{34}$ R. Y. Dong, J. Magn. Reson. 66, 422 (1986)

${ }^{35}$ R. Y. Dong, J. Chem. Phys. 88(6), 3962 (1988).

${ }^{36}$ L. Beguin, J. W. Emsley, M. Lelli, A. Lesage, G. R. Luckhurst, B. A. Timimi, and H. Zimmermann, J. Phys. Chem. B 116, 7940 (2012).

${ }^{37}$ A. C. J. Weber, A. Pizzirusso, L. Muccioli, C. Zannoni, W. L. Meerts, C. A. de Lange, and E. E. Burnell, J. Chem. Phys. 136, 174506 (2012).

${ }^{38}$ A. C. J. Weber, E. E. Burnell, W. L. Meerts, C. A. de Lange, R. Y. Dong, L. Muccioli, A. Pizzirusso, and C. Zannoni, J. Chem. Phys. 143, 011103 (2015).

${ }^{39}$ E. E. Burnell, A. C. J. Weber, R. Y. Dong, W. L. Meerts, and C. A. de Lange, J. Chem. Phys. 142, 024904 (2015).

${ }^{40}$ I. D. Campbell and R. Freeman, J. Magn. Reson. 11, 143 (1973).

${ }^{41}$ A. Kalk and H. J. C. Berendsen, J. Magn. Reson. 24, 343 (1976).

${ }^{42}$ Q. R. Passey, K. Bohacs, W. L. Esch, R. Klimentidis, and S. Sinha, in International Oil and Gas Conference and Exhibition in China, 8-10 June, Beijing, China (Society of Petroleum Engineers, 2010), SPE-131350-MS.

${ }^{43}$ R. G. Loucks, R. M. Reed, S. C. Ruppel, and U. Hammes, AAPG Bull. 96(6), 1071 (2012).

${ }^{44}$ P. M. Singer, Z. Chen, and G. J. Hirasaki, Petrophysics 57(6), 604 (2016).

${ }^{45}$ K. E. Washburn and Y. Cheng, J. Magn. Reson. 278, 18 (2017).

${ }^{46}$ A. E. Ozen and R. Sigal, Petrophysics 54(1), 11 (2013).

${ }^{47}$ R. E. Lewis, P. M. Singer, T. Jiang, E. Rylander, S. M. Sinclair, and R. H. McLin, in SPE Unconventional Resources Conference-USA, 10-12 April, The Woodlands, Texas, USA (Society of Petroleum Engineers, 2013), SPE164554-MS.
${ }^{48}$ T. Jiang, E. Rylander, P. M. Singer, R. E. Lewis, and S. M. Sinclair, in SPWLA 54th Annual Logging Symposium, 22-26 June, New Orleans, Louisiana (Society of Petrophysicists and Well-Log Analysts, 2013), SPWLA-2013-LL.

${ }^{49}$ P. M. Singer, E. Rylander, T. Jiang, R. McLin, R. E. Lewis, and S. M. Sinclair, Society of Core Analysts SCA2013-18 (2013).

${ }^{50}$ K. E. Washburn and J. E. Birdwell, J. Magn. Reson. 233, 17 (2013).

${ }^{51}$ R. Kausik, K. Fellah, E. Rylander, P. M. Singer, R. E. Lewis, and S. M. Sinclair, Society of Core Analysts SCA2014-73 (2014).

${ }^{52} \mathrm{H}$. Daigle, A. Johnson, J. P. Gips, and M. Sharma, in Unconventional Resources Technology Conference, 25-27 August, Denver, Colorado, USA (Unconventional Resources Technology Conference, 2014), URTEC-1905272-MS.

${ }^{53}$ K. E. Washburn, Concepts Magn. Reson., Part A 43A(3), 57 (2014).

${ }^{54}$ J.-P. Korb, B. Nicot, A. Louis-Joseph, S. Bubici, and G. Ferrante, J. Phys. Chem. C 118(40), 23212 (2014).

${ }^{55}$ B. Nicot, N. Vorapalawut, B. Rousseau, L. F. Madariaga, G. Hamon, and J.-P. Korb, Petrophysics 57(1), 19 (2015).

${ }^{56}$ M. Lessenger, R. Merkel, R. Medina, S. Ramakrishna, S. Chen, R. Balliet, H. Xie, P. Bhattad, A. Carnerup, and M. Knackstedt, in SPWLA 56th Annual Logging Symposium, 18-22 July, Long Beach, California, USA (Society of Petrophysicists and Well-Log Analysts, 2015), SPWLA-2015-UUU.

${ }^{57}$ J. E. Birdwell and K. E. Washburn, Energy Fuels 29, 2234 (2015).

${ }^{58}$ M. Fleury and M. Romero-Sarmiento, J. Pet. Sci. Eng. 137, 55 (2016).

${ }^{59}$ R. Kausik, K. Fellah, E. Rylander, P. M. Singer, R. E. Lewis, and S. M Sinclair, Petrophysics 57(4), 339 (2016).

${ }^{60}$ B. Sun, E. Yang, H. Wang, S. J. Seltzer, V. Montoya, J. Crowe, and T. Malizia, in SPWLA 57th Annual Logging Symposium, 25-29 June, Reykjavik, Iceland (Society of Petrophysicists and Well-Log Analysts, 2016), SPWLA-2016PP.

${ }^{61}$ C. Sondergeld, A. Tinni, C. Rai, and A. Besov, in SPWLA 57th Annual Logging Symposium, 25-29 June, Reykjavik, Iceland (Society of Petrophysicists and Well-Log Analysts, 2016), SPWLA-2016-RRR.

${ }^{62}$ D. Yang and R. Kausik, Energy Fuels 30(6), 4509 (2016).

${ }^{63}$ Z. Chen, P. M. Singer, J. Kuang, M. Vargas, and G. J. Hirasaki, Petrophysics 58(5), 470 (2017).

${ }^{64}$ A. Valori, S. V. den Berg, F. Ali, and W. Abdallah, Energy Fuels 31, 5913 (2017).

${ }^{65}$ J. Liu, L. Wang, S. Xi, D. Asthagiri, and W. G. Chapman, Langmuir 33(42), 11189 (2017).

${ }^{66}$ J. C. Phillips, R. Braun, W. Wang, E. Tajkhorshid, E. Villa, C. Chipot, R. Skeel, L. Kale, and K. Schulten, J. Comput. Chem. 26, 1781 (2005).

${ }^{67}$ K. Vanommeslaeghe, E. Hatcher, C. Acharya, S. Kundu, S. Zhong, J. Shim, E. Darian, O. Guvench, P. Lopes, I. Vorobyov, and A. D. Mackerell, Jr., J. Comput. Chem. 31, 671 (2010).

${ }^{68}$ L. Martinez, R. Andrade, E. G. Birgin, and J. M. Martinez, J. Comput. Chem. 30, 2157 (2009).

${ }^{69}$ S. Plimpton, J. Comput. Phys. 117, 1 (1995).

${ }^{70}$ P. J. in't Veld and G. C. Rutledge, Macromolecules 36, 7358 (2003).

${ }^{71} \mathrm{~J}$. McConnell, The Theory of Nuclear Magnetic Relaxation in Liquids (Cambridge University Press, 1987)

${ }^{72}$ R. Kimmich, NMR Tomography, Diffusometry and Relaxometry (SpringerVerlag, 1997)

${ }^{73}$ L. Venkataramanan, Y.-Q. Song, and M. D. Hürlimann, IEEE Trans. Signal Process. 50(5), 1017 (2002).

${ }^{74}$ Y.-Q. Song, L. Venkataramanan, M. D. Hürlimann, M. Flaum, P. Frulla, and C. Straley, J. Magn. Reson. 154, 261 (2002).

${ }^{75}$ Y. Ayant, E. Belorizky, J. Aluzon, and J. Gallice, J. Phys. 36(10), 991 (1975)

${ }^{76}$ Y. Ayant, E. Belorizky, P. Fries, and J. Rosset, J. Phys. 38(3), 325 (1975).

${ }^{77}$ I. Solomon, Phys. Rev. 99(2), 559 (1955).

${ }^{78}$ H. T. Edzes and E. T. Samulski, Nature 265, 521 (1977).

${ }^{79}$ J. B. Lambert and E. P. Mazzola, Nuclear Magnetic Resonance Spectroscopy: An Introduction to Principles, Applications, and Experimental Methods (Prentice-Hall, 2004). 